\title{
Left ventricular outflow tract pseudoaneurysm diagnosed with point-of-care ultrasound in the emergency department
}

\author{
Puneet Kapur, MSc, MD*; Melanie Baimel, $\mathrm{MD}^{\dagger \neq}$; Jordan Chenkin, MD, MEd ${ }^{\dagger \ddagger}$
}

\section{ABSTRACT}

Left ventricular outflow tract pseudoaneurysms are a rare but lifethreatening disorder, often caused by complications of cardiac surgery or myocardial infarction. We present a case report of a patient with no prior risk factors who presented with a six-month history of progressive exertional dyspnea, bilateral leg swelling and cough. Point-of-care ultrasound revealed an unexpected outpouching of the left ventricle. He was diagnosed with a left ventricular outflow tract pseudoaneurysm and subsequently went into cardiogenic shock secondary to extension of pseudoaneurysm causing extrinsic compression of the coronary arteries. The patient underwent successful emergency surgical repair and made a full recovery.

\section{RÉSUMÉ}

Les faux anévrysmes de la chambre de chasse du ventricule gauche sont une affection rare mais potentiellement mortelle, résultant souvent de complications de la chirurgie cardiaque ou d'un infarctus du myocarde. Sera exposé ici le cas d'un patient sans facteurs de risque antérieurs, qui a consulté pour la présence d'une dyspnée progressive à l'effort, d'œdème des membres inférieurs et de toux depuis six mois. L'échographie au lieu d'intervention a révélé l'existence inattendue d'un bombement du ventricule gauche. Un diagnostic de faux anévrysme de la chambre de chasse du ventricule gauche a été posé et, plus tard, le patient est passé en état de choc cardiogène, consécutif au prolongement du faux anévrysme, ce qui a causé une compression extrinsèque des artères coronaires. Le patient a subi une réparation chirurgicale d'urgence couronnée de succès, et il s'est rétabli complètement.

Keywords: ultrasonography, pseudoaneurysm, POCUS, LVOT, left ventricular outflow tract

\section{CASE REPORT}

A 28-year-old male presented to the acute care section of the emergency department (ED) complaining of a 6-month history of epigastric pain and dyspepsia, as well as unintentional weight loss of nine kilograms. In addition, over the last three months, he had developed a non-productive cough, exertional dyspnea, and bilateral leg swelling. His past medical history was unremarkable other than previous treatment for Helicobacter pylori. He denied any history of smoking or intravenous drug abuse. He had several visits to primary care physicians for his symptoms and was diagnosed with community-acquired pneumonia, for which he was given a prescription for antibiotics without improvement. When he finally presented to the ED, he expressed worsening exertional dyspnea and decreased urine output over several days despite adequate hydration.

On examination, the patient appeared well and was in no acute distress. His vitals were as follows: pulse rate 117 beats per minute, blood pressure 124/85 mm Hg, respiratory rate of 20 breaths per minute, oxygen saturation of $96 \%$ on room air, and temperature of $37.2^{\circ} \mathrm{C}\left(98.96^{\circ} \mathrm{F}\right)$. A respiratory exam was normal with clear breath sounds bilaterally. A cardiovascular exam showed a jugular venous pressure $6 \mathrm{~cm}$ above the sternal angle and a bilateral $1+$ pitting edema up to the knees. Auscultation revealed a grade $3 / 6$ holosystolic murmur at the apex radiating to the axilla at the left lower sternal border and a crescendo-decrescendo murmur at the upper sternal border that radiated to the clavicle.

Investigations included a plain chest radiograph that demonstrated no focal consolidation, pulmonary edema, or pleural effusions. The initial electrocardiogram (ECG) rhythm was significant for sinus tachycardia with no ischemic changes. Laboratory investigations including high sensitivity troponin were normal, except for slightly elevated creatinine $(166 \mu \mathrm{mol} / \mathrm{L})$, alanine transaminase (ALT; 204U/L), and aspartate transaminase (AST; 157 U/L) levels. The patient underwent a computed tomography (CT) pulmonary angiogram, which revealed

From the *Department of Emergency Medicine, University of Saskatchewan, Saskatoon, SK; †Sunnybrook Health Sciences Centre, Toronto, ON; and the $¥$ Division of Emergency Medicine, University of Toronto, Toronto, ON

Correspondence to: Dr. Puneet Kapur, Box 16, 103 Hospital Drive, Saskatoon, SK, S7N 0W8; Email: Puneet@Kapur.Org 
no evidence of a pulmonary embolism and a normal heart, except for a prominent left atrial appendage. Based on clinical features of volume overload and audible murmurs with no known history or risk factors for coronary artery or valvular disease, we proceeded with a cardiac point-of-care ultrasound (POCUS) examination. On the parasternal long axis and apical four-chamber views, there was an abnormal ballooning of the left ventricular outflow tract (LVOT), concerning for a left ventricular outflow tract pseudoaneurysm (LVOTPA) (Figure 1 and 2 and Supplementary Video 1 and 2).

Based on this POCUS finding, the cardiology service was consulted for further investigation. A transesophageal echocardiogram confirmed a pseudoaneurysm of the LVOT measuring $5.0 \mathrm{~cm} \times 2.1 \mathrm{~cm}$, with a neck measuring $1.3 \mathrm{~cm}$. The LVOTPA had ruptured, resulting in communication with the left atrium, severe mitral regurgitation, and mild aortic regurgitation. The patient was admitted to the coronary care unit for further investigation and possible surgical repair. During the inpatient admission, he developed severe chest pain with diffuse ST-segment depression and ST elevation in aVR. Subsequently, he underwent an emergent echocardiogram and cardiac catheterization and was diagnosed with cardiogenic shock secondary to extension of the LVOTPA, with severe extrinsic compression of the left main and circumflex arteries. Cardiovascular surgery was consulted, and the patient was taken to the operating room emergently for successful repair of the pseudoaneurysm. Serology and

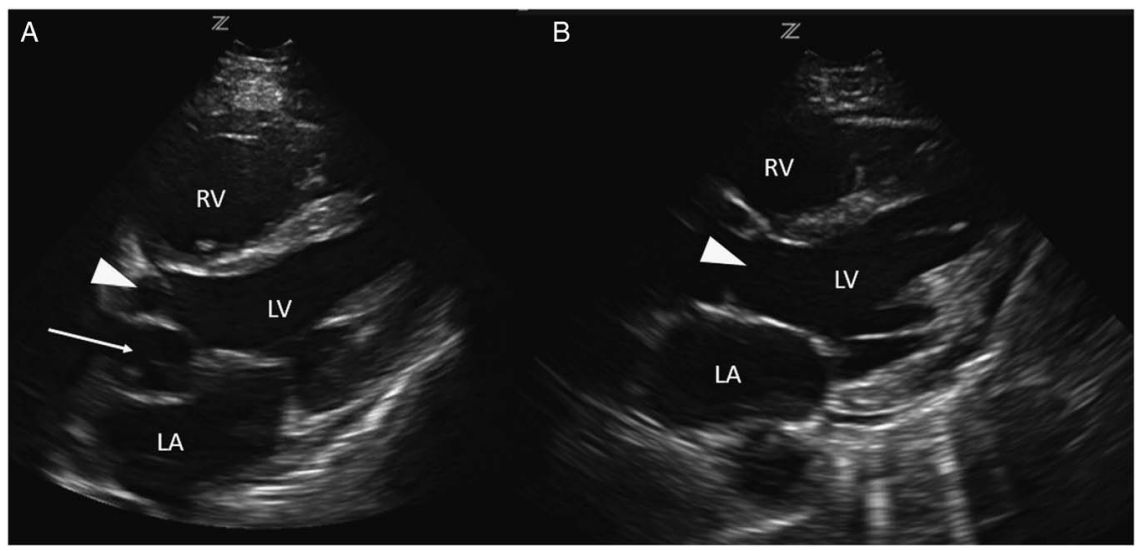

Figure 1. A comparison of this patient's parasternal long axis view $(A)$ with that of a normal patient (B), demonstrating the presence of a ballooning area compressing the left atrium caused by a pseudoaneurysm (arrow) adjacent to the LVOT (arrowhead). LV = left ventricle; RV = right ventricle; LA = left atrium. Note that the image is using a radiological orientation, rather than a traditional cardiology orientation.

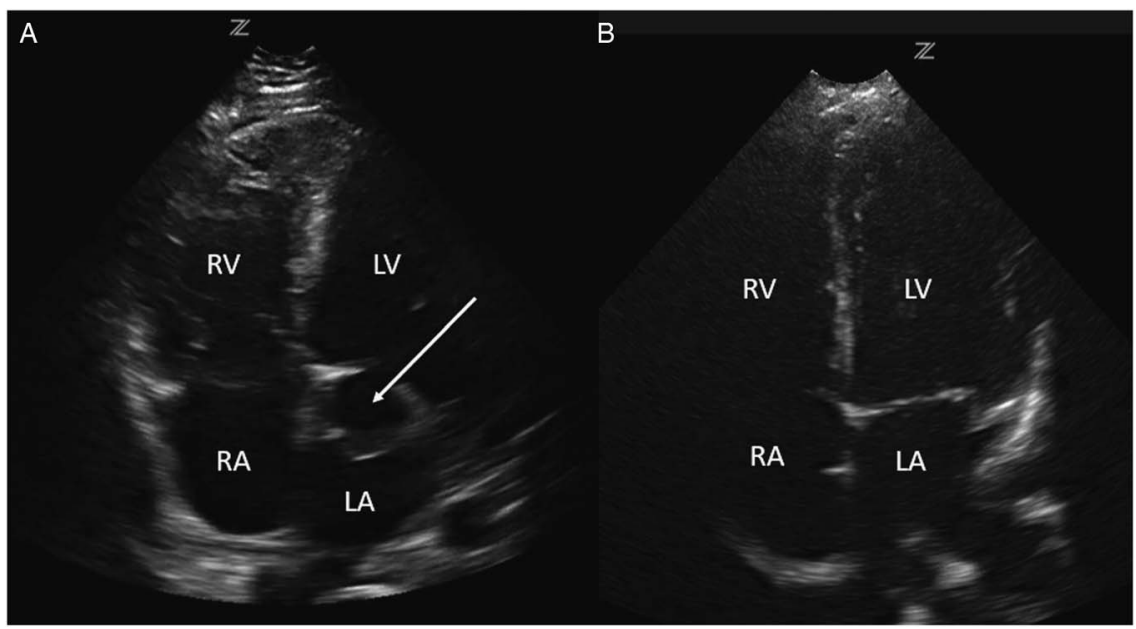

Figure 2. A comparison of this patient's apical four-chamber view (A) with that of a normal patient (B), demonstrating the presence of an aneurysmal dilation of the LVOT compressing the left atrium (arrow). 
pathological samples were all negative for an infectious cause of the pseudoaneurysm. The patient was discharged 25 days later and has continued to do well during follow-up. Subsequent investigations with specialists in infectious diseases and rheumatology failed to identify an infectious or connective tissue diagnosis, and the cause of the LVOTPA in this patient remains unknown.

\section{DISCUSSION}

LVOTPA is a rare but deadly disorder that can present with non-specific signs and symptoms. The exact incidence and prevalence of this disorder are unknown, as only case reports exist in the literature. ${ }^{1}$ An LVOTPA is defined as an outpouching of the LVOT, with blood contained only by a thickened epicardial or pericardial layer. The high morbidity and mortality associated with this condition is related to its proximity to the aortic root and the high risk of rupture. ${ }^{2}$

The low incidence of disease and variable clinical presentations of LVOTPA pose a diagnostic challenge. Risk factors include a history of cardiac surgery, infective endocarditis, chest trauma, or myocardial infarction, ${ }^{3,4}$ although there is a case report of an idiopathic presentation..$^{5}$ Symptoms are generally caused by the pseudoaneurysm sac compressing the surrounding structures such as the coronary arteries, pulmonary artery, LVOT, left atrium, and mainstem bronchus. ${ }^{6}$ Common presentations include heart failure, chest pain, and dyspnea. However, it is not uncommon for patients to present with non-specific complaints such as abdominal pain, cough, and dizziness, and over $10 \%$ can be asymptomatic. ${ }^{3,7}$ Physical examination findings are often non-specific but can include signs of heart failure, mitral regurgitation, and aortic insufficiency murmur, as well as a unique to-and-fro murmur because of turbulent flow across a narrow aneurysmal neck. ${ }^{8,9}$ An ECG may show ST-segment elevation, Q waves, or non-specific ST abnormalities. ${ }^{8}$ Chest $\mathrm{x}$-rays are similarly non-specific but can show enlargement of the cardiac border or pulmonary edema. ${ }^{3}$ A lack of specific historical features, physical exam findings, or laboratory findings is why echocardiography is essential for diagnosis.

Emergency physicians are increasingly using POCUS as an adjunct to a physical examination for patients presenting with shortness of breath, chest pain, and undifferentiated shock. Studies have demonstrated that emergency physicians can use POCUS to identify important cardiac abnormalities such as left ventricular systolic dysfunction, pericardial effusions, and right ventricular dilation. ${ }^{10-12}$ Cardiac POCUS can be a valuable adjunct to the physical examination for determining the cause of shock in critically ill patients and is an important component of ultrasound resuscitation protocols such as the Rapid Ultrasonography for Shock and Hypotension (RUSH) protocol. ${ }^{13}$ As emergency physicians become more experienced with cardiac POCUS, more advanced applications are being investigated such as valvular assessment, regional wall motion, and diastolic function. ${ }^{14-16}$

As a consequence of this increased experience with cardiac POCUS, emergency physicians may be able to identify if the heart does not appear normal because of other important cardiac abnormalities. In this case, the emergency physician identified a structural cardiac abnormality using POCUS that led to the request for an expedited inpatient workup. The primary finding of an LVOTPA with echocardiography is an abnormal outpouching in the LVOT. This may manifest as a discontinuity in the myocardial border ${ }^{17}$ or the detection of an extra cavity beyond the normal four cardiac chambers (Figure 1 and 2). ${ }^{18}$ Once an abnormal left ventricular outpouching is identified, a transesophageal echocardiogram should be ordered to confirm the diagnosis, to determine the extent of the aneurysm, and to determine if there is any impact on surrounding structures.

Patients with suspected LVOTPAs should generally be referred for inpatient workup. Surgical resection is the treatment of choice for LVOTPAs, with a few exceptions. Unlike true aneurysms that can be managed with elective surgery, up to $30 \%-45 \%$ of pseudoaneurysms will spontaneously rupture, and mortality post-rupture approaches $100 \% .{ }^{18}$ In some rare cases in which an LVOTPA has been present for an extended period (>3 months) ${ }^{6}$ or in asymptomatic patients with small aneurysms $(<3 \mathrm{~cm})$, medical management may be an option. ${ }^{19,20}$ For emergency physicians, the suspected diagnosis of an LVOTPA should prompt immediate subspecialty consultation given the high mortality rates with rupture.

\section{CONCLUSION}

Emergency physicians are increasingly using cardiac POCUS to help identify important life-threatening conditions and guide management in the ED. Through this 
increased experience, emergency physicians may also be able to recognize if the anatomy appears structurally abnormal and seek further testing. In the case presented, the use of POCUS helped to identify a structural cardiac abnormality that was contributing to the patient's presentation. This led to an expedited inpatient workup and diagnosis of a rare condition that ultimately required emergency surgical repair in a situation in which a delay for outpatient evaluation might have had a fatal outcome. The appearance of an outpouching in the LVOT should prompt emergency physicians to consider an LVOTPA and obtain emergency cardiology consultation in the ED.

\section{Competing interests: None}

\section{SUPPLEMENTARY MATERIAL}

To view supplementary material for this article, please visit https://doi.org/10.1017/cem.2018.20

\section{REFERENCES}

1. Kharwar RB, Shukla A. Huge multilobulated left ventricular outflow tract pseudoaneurysm presenting with ventricular tachycardia. 7 Cardiovasc Ultrasound 2015;23(4):276-9.

2. Goldfarb B, Rovner M, Goldbraich N. Pseudoaneurysm of the left ventricular outflow tract with reentry into the ascending aorta: an iatrogenic left ventricular ascending aortic fistula. 7 Am Soc Echocardiogr 1997;10(8):866-8.

3. Frances C, Romero A, Grady D. Left ventricular pseudoaneurysm. 7 Am Coll Cardiol 1998;32(3):557-61.

4. Yeo TC, Malouf JF, Oh JK, Seward JB. Clinical profile and outcome in 52 patients with cardiac pseudoaneurysm. Ann Intern Med 1998;128(4):299-305.

5. Jha AK, Pandey R, Gharde P, Devagourou V, Kiran U. Idiopathic left ventricular outflow tract pseudoaneurysm. Ann Card Anaesth 2013;16(3):209-11.

6. Yeo TC, Malouf JF, Reeder GS, Oh JK. Clinical characteristics and outcome in postinfarction pseudoaneurysm. Am 7 Cardiol 1999;84(5):592-5.

7. Narvaez Muñoz AF, Gonzalez Cambeiro C, Duran Muñoz D, Rosario Ortiz A. Giant left ventricular pseudoaneurysm manifested with a persistent cough. 7 Card Surg 2017; 32(5):285-7.

8. Cho MN, Mehta SK, Matulevicius S, et al. Differentiating true versus pseudo left ventricular aneurysm: a case report and review of diagnostic strategies. Cardiol Rev 2006;14(6): e27-30.

9. Sutherland GR, Smyllie JH, Roelandt JR. Advantages of colour flow imaging in the diagnosis of left ventricular pseudoaneurysm. Br Heart 7 1989;61(1):59-64.

10. McKaigney CJ, Krantz MJ, La Rocque CL, et al. E-point septal separation: a bedside tool for emergency physician assessment of left ventricular ejection fraction. Am 7 Emerg Med 2014;32(6):493-7.

11. Dresden S, Mitchell P, Rahimi L, et al. Right ventricular dilatation on bedside echocardiography performed by emergency physicians aids in the diagnosis of pulmonary embolism. Ann Emerg Med 2014;63(1):16-24.

12. Seif D, Perera P, Mailhot T, Riley D, Mandavia D. Bedside ultrasound in resuscitation and the rapid ultrasound in shock protocol. Crit Care Res Pract 2012;2012:503254.

13. Perera P, Mailhot T, Riley D, Mandavia D. The RUSH exam: rapid Ultrasound in SHock in the evaluation of the critically 1ll. Emerg Med Clin North Am 2010;28(1): 29-56.

14. Lebeau R, Sas G, El Rayes M, et al. Left ventricular ejection fraction assessment by non-cardiologists from transverse views using a simplified wall motion score index. Echo Res Pract 2015;2(1):1-8.

15. Alzahrani H, Woo MY, Johnson C, Pageau P, Millington S, Thiruganasambandamoorthy V. Can severe aortic stenosis be identified by emergency physicians when interpreting a simplified two-view echocardiogram obtained by trained echocardiographers? Crit Ultrasound 7 2015;7(1):5.

16. Ehrman RR, Russell FM, Ansari A, et al. Can emergency physicians accurately diagnose and correctly classify diastolic dysfunction using bedside echocardiography? Acad Emerg Med 2014;21:S327.

17. Zoffoli G, Mangino D, Venturini A, et al. Diagnosing left ventricular aneurysm from pseudo-aneurysm: a case report and a review in literature. 7 Cardiothorac Surg 2009;4(1):11.

18. Bisoyi S, Dash AK, Nayak D, Sahoo S, Mohapatra R. Left ventricular pseudoaneurysm versus aneurysm a diagnosis dilemma. Ann Card Anaesth 2016;19(1):169-72.

19. Prêtre R, Linka A, Jenni R, Turina MI. Surgical treatment of acquired left ventricular pseudoaneurysms. Ann Thorac Surg 2000;70(2):553-7.

20. Moreno R, Gordillo E, Zamorano J, et al. Long term outcome of patients with postinfarction left ventricular pseudoaneurysm. Heart 2003;89(10):1144-6. 